“(C) 2015 IEEE. Personal use of this material is permitted. Permission from IEEE must be obtained for all other uses, in any current or future media, including reprinting/republishing this material for advertising or promotional purposes, creating new collective works, for resale or redistribution to servers or lists, or reuse of any copyrighted component of this work in other works." 


\title{
Modeling Technological Topic Changes in Patent Claims
}

\author{
Hongshu Chen ${ }^{12}$, Yi Zhang ${ }^{12}$, Guangquan Zhang ${ }^{1}$, Donghua Zhu ${ }^{2}$, Jie Lu ${ }^{1}$ \\ ${ }^{1}$ Decision Systems \& e-Service Intelligence Lab, Centre for Quantum Computation \& Intelligent Systems \\ Faculty of Engineering and Information Technology, University of Technology Sydney, Sydney, Australia \\ ${ }^{2}$ School of Management and Economics, Beijing Institute of Technology, Beijing, China
}

\begin{abstract}
Patent claims usually embody the most essential terms and the core technological scope to define the protection of an invention, which makes them the ideal resource for patent content and topic change analysis. However, manually conducting content analysis on massive technical terms is very time consuming and laborious. Even with the help of traditional text mining techniques, it is still difficult to model topic changes over time, because single keywords alone are usually too general or ambiguous to represent a concept. Moreover, term frequency which used to define a topic cannot separate polysemous words that are actually describing a different theme. To address this issue, this research proposes a topic change identification approach based on Latent Dirichlet Allocation to model and analyze topic changes with minimal human intervention. After textual data cleaning, underlying semantic topics hidden in large archives of patent claims are revealed automatically. Concepts are defined by probability distributions over words instead of term frequency, so that polysemy is allowed. A case study using patents published in the United States Patent and Trademark Office (USPTO) from 2009 to 2013 with Australia as their assignee country is presented to demonstrate the validity of the proposed topic change identification approach. The experimental result shows that the proposed approach can be used as an automatic tool to provide machine-identified topic changes for more efficient and effective R\&D management assistance.
\end{abstract}

\section{INTRODUCTION}

Patent claims, as an important part of unstructured segments of a patent document, hold explicit information and implicit knowledge revealing technological concepts, topics and related R\&D activities with concise, but precise language $[1,2]$. It is often argued as a valuable source for the detection of technological changes and to gain technological insight [35]. Since manually conducting content analysis on massive patent documents is very time-consuming and laborious, in recent years, one of the fundamental changes to research in $R \& D$ management is the access to extremely powerful information techniques and a vast amount of digital and textual data [6]. In particular, for efficient patent analysis, automatic approaches to assist domain experts and decision makers to mine and understand large volumes of patent documents have drawn increasing attention and still are in great demand [7]

Much effort has been devoted to reveal latent knowledge from the textual data of patent documents. Watts and Porter [8] suggested an approach to investigate terminological trends by tracking the historical change of keywords. Yoon and Park [9] presented a keyword-based morphology study to identify the detailed configurations of promising technology.
Zhang and his colleague [10] introduced a term clumping approach based on Principal Components Analysis to explore keywords and main phrases in abstract from scientific literature. In addition, text analytics have already been applied to Technology Intelligence application TrendPerceptor [11], Techpioneer [12],VantagePoint [13] and Aureka [14] to determine hidden concepts and relationships, where clustering, classification and mapping techniques were used to support further content analysis of technological documents. However, before most of these applications are applied, several sets of keywords need to be defined in advance, which still derive from the opinion and knowledge of domain experts. Moreover, the outcomes of traditional text mining techniques are single keywords with ranking, yet these words alone are usually too general or misleading for indicating a concept, especially when there are polysemous words actually describing different topics [7].

To overcome these limitations, this research proposes a topic change identification approach using Latent Dirichlet Allocation. Unsupervised topic modeling is applied to vast amounts of target patent claims, providing a corpus structure with minimal human intervention. There is no pre-set classification or keywords list for this approach and the results are discovered in a completely unsupervised way. In addition, instead of using single terms, topics are represented by probability distributions over words. The actual semantic meaning of a topic is able to be delivered in this way, and at the same time the polysemous words which are actually depicting different concepts, can also be separated. After revealing topics from patent sub-collections of different years, a topic change model is utilized to identify topic changes over time. Finally, to demonstrate the performance of our proposed approach, patents published during years 2009 to year 2013 in the United States Patent and Trademark Office (USPTO) with Australia as their assignee country, are selected to present a case study. The experimental result demonstrates that the proposed approach is able to provide machine-identified topic changes automatically without any pre-setting of keywords. The outcomes of our approach will be used to serve R\&D management assistance.

This paper is organized as follows: Section II reviews related research developments by introducing patent data in technological research and Latent Dirichlet Allocation. Section III describes the proposed topic change identification approach step by step. Section IV carries out experiments using USPTO patents to demonstrate the proposed approach in a real patent analysis context. The conclusions and future study are addressed in Section V. 


\section{LITERATURE REVIEW}

\section{A. Patent Data in Technological Research}

Patent documents are composed of structured information and unstructured descriptions of inventions. Analytical approaches based on structured data of patents, such as issue date, inventor, assignees or International Patent Classification, have played the major role in both theoretical and practical research [15-17]. However, the unstructured data in patent documents, such as abstracts, claims, and descriptions usually contain much more abundant information than the structured sections, since they contain significant characteristics, detailed functionalities, or major contributions of technologies. Therefore, in recent years, there has been a lot of interest in applying text mining techniques to unstructured patent data to set domain analysts free from studying and understanding massive amounts of technological content [7, 18].

Patent claims, as an important part of unstructured segments of a patent document, embody all the important technical features of an invention with the most essential technological terms to define the protection [19]. A patent claim usually consists of three parts: a Preamble that serves as an introductory section to recite the primary purpose, function or properties; a transition phrase, such as comprising, having including, consisting of, etc.; a 'body' that contains the elements or steps that together describe the invention [2022]. Claims, on one hand, reveal the core inventive topics and the major technological scope of a patent; on the other hand, they are written in concise, but precise language, which make them the best resource for identifying technological topics and facilitating patent document analysis [1, 2, 20, 23]. This research utilizes patent claims as the main source of topic change analysis.

Among patent databases from different countries, the United States Patent and Trademark Office (USPTO) database is mostly used because patents submitted in other countries are often also simultaneously submitted in the United States [24].

\section{B. Latent Dirichlet Allocation}

Latent Dirichlet Allocation (LDA) [25] is a probabilistic model that aims to estimate the properties of multinomial observations by unsupervised learning. It gives an estimation of the latent semantic topics hidden in large archives of documents, and indicates the probabilities of how various documents belong to different topics. LDA has been used as a very efficient tool to assist topic discovery and analysis, in practice. For example, Griffiths and Steyvers [26] applied LDA-based topic modeling to discover the hot topics covered by papers in Proceedings of the National Academy of Sciences of the United States of America (PNAS); Yang et al. [27] proposed a Topic Expertise Model (TEM) based on LDA to jointly model topics and expertise for Community Question Answering (CQA) with Stack Overflow data; Kim and $\mathrm{Oh}$ [28] proposed a framework based on LDA to identify important topics and their meaningful structure within the news archives on the Web.

The graphical model of LDA is presented in Fig. 1, showing three rectangular plates where: $D$ denotes the overall documents in a corpus; $K$ indicates the topic numbers for $D$; and $N_{d}$ stands for the term number of $d^{\text {th }}$ document in document collection $D$. Each node in Fig. 1 stands for a random variable in the generative process of LDA. All the plates in the figure indicate replication. On the left of the figure, $\vec{\vartheta}_{d}$ stands for the topic proportions for the $d^{\text {th }}$ document. For document $d$, the topic assignments are $Z_{d}$, where $Z_{d, n}$ indicates the topic assignment of the $n^{\text {th }}$ word in the $d^{\text {th }}$ document. On the right of the figure, the topics themselves are illustrated by $\vec{\varphi}_{1: K}$, where each $\vec{\varphi}_{k}$ is a distribution over vocabularies. All of the unshaded circles indicate hidden nodes. The shaded circles are observable nodes, where $W_{d, n}$ stands for the $n^{\text {th }}$ word in document $d$. Finally, $\alpha$ and $\beta$ are two hyperparameters that determine the amount of smoothing applied to the topic distributions for each document and the word distributions for each topic [25, 29-31]. In summary, the generative process of LDA can be denoted by the joint distribution of the random variables in Fig. 1 .

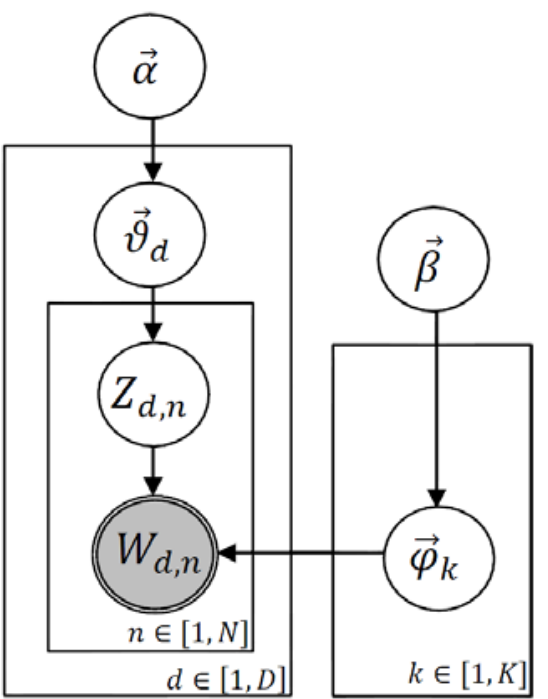

Fig 2. The graphical model of Latent Dirichlet Allocation

The parameters of LDA need to be estimated by an iterative approach. Among existing approaches, Gibbs sampling, which is one of the most commonly used methods, is an approximate inference algorithm based on the Markov Chain Monte Carlo (MCMC) and widely used to estimate the assignment of words to topics by observed data [26, 32, 33]. The randomness introduced by the initiation of the sampling affects the estimation of probabilities in LDA, so that the result is slightly different even with exactly the same setting of input and parameters; yet on the whole, the results of different experiments won't change much. 


\section{METHODOLOGY}

This section explains the details of our proposed topic change identification approach. The framework is given first, then each detailed step is illustrated.

\section{A. Framework}

The overall framework of our proposed topic change identification approach is shown in Fig. 2. Users first initiate search statements to declare their domain analytic needs. Patent ID, title, claims, issue time, assignees, United States Patent Classification (USPC) and other information of target patents are then crawled into a database waiting for further analysis. To identify topic changes over time, the whole patent collection is first divided into several sub-collections and labeled with their corresponding issue year. Subsequently, for each sub-collection, patent claims and titles embodying essential technical terms, and USPC providing a general understanding of the domain classification are extracted from the target patents database. The two plates in the figure indicate replication.

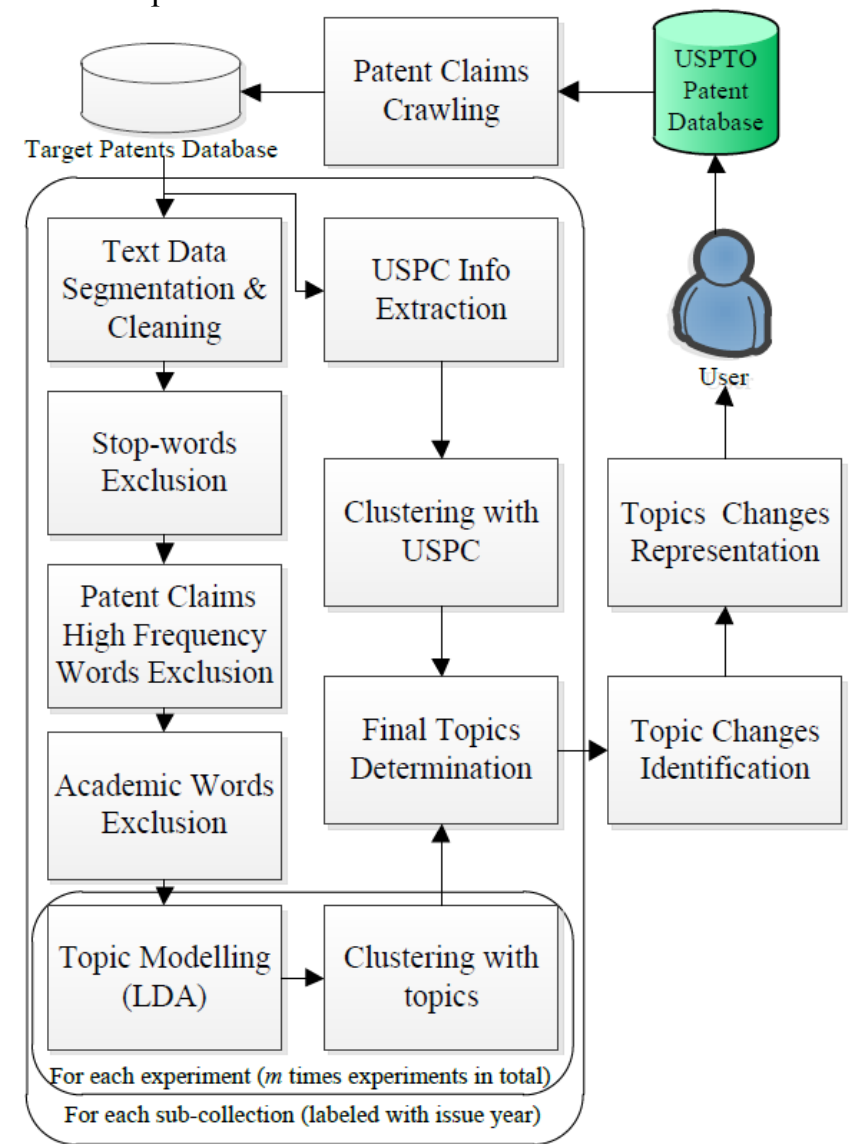

Fig. 2 The framework of the proposed topic change identification approach

Textual data composed by claims and titles, after data segmentation and cleaning, are then placed into a series of words exclusion modules to filter out the most common function words, high frequency words that commonly appeared in patent claims, and academic words with vague and general meanings. Then, the prepared text will be passed to the topic modeling module. Meanwhile, the USPC information of the corresponding patents is extracted to assist final topic determination. As mentioned, the randomness introduced by the initiation of the sampling will affect the result of LDA. To acquire the most reliable topics of the corpus, we utilize USPC as a measurement to evaluate results from $m$ times experiments. Patents are clustered with both their USPC and topic assignments. The final topic modeling result is the one that provides the most similar clusters to the USPC clustering outcome. Finally, with all the topics estimated from patent sub-collections of different years, topic changes over time can be identified and presented to users.

\section{B. Patent Claim Text Cleaning}

Patent claims are a special kind of textual data that contain plenty of technical terms, specific words serving as transition phrases and numerous academic words that describe invention outcomes. Among all the terms that one claim may contain, only technical terms provide most meaningful information reflecting technological topics. Therefore, for patent collections of each year, as shown in Fig. 2, before modeling topics with LDA, we utilize three modules to remove general words from the corpus of patents as follows:

- Stop words such as the, that, these

- High frequency words in patent claims such as claimed, comprising, invention

- General academic words such as research, approach, data.

The stop words list we applied is from an information retrieval Resources link from Stanford University [34]; the patent claim commonly used phrases are summarized from a Transitional Phrase page on Wikipedia [35]; the general academic words list is provided by the University of Nottingham, we select the top 100 most frequent academic words and remove them from our final corpus $[10,36]$.

\section{Topic Modeling}

LDA utilizes a probability distribution over words, instead of a single term, to define a concept, delivering the semantic meaning of the topic and, at the same time, allowing polysemy. Thus it is very suitable for "understanding" the content of large corpuses such as emails, news, scientific papers and our main data source here, patent claims. After removing all commonly used words from the corpus, we utilize LDA to generate several groups of topics for a number of patent sub-collections, which are labeled by their corresponding issue year. In a sub-collection of the corpus, the claims and title of each patent constitute one document, and the number of documents equals the number of patents; the USPC and other structural information are stored alone in a single file to assist further topic determination. All the textual documents in the corpus are seen as mixtures of a number of topics; each topic is seen as a distribution over various vocabularies. Here we present the global topics 
as $\vec{P}_{1: t}=\left(\vec{P}_{1}, \vec{P}_{2}, \ldots, \vec{P}_{i}, \ldots, \vec{P}_{t}\right)$, where $\vec{P}_{i}$ stand for the topics of the $i^{\text {th }}$ sub-collection of the corpus. The relationship between sub-collections and topics is illustrated in Fig. 3.

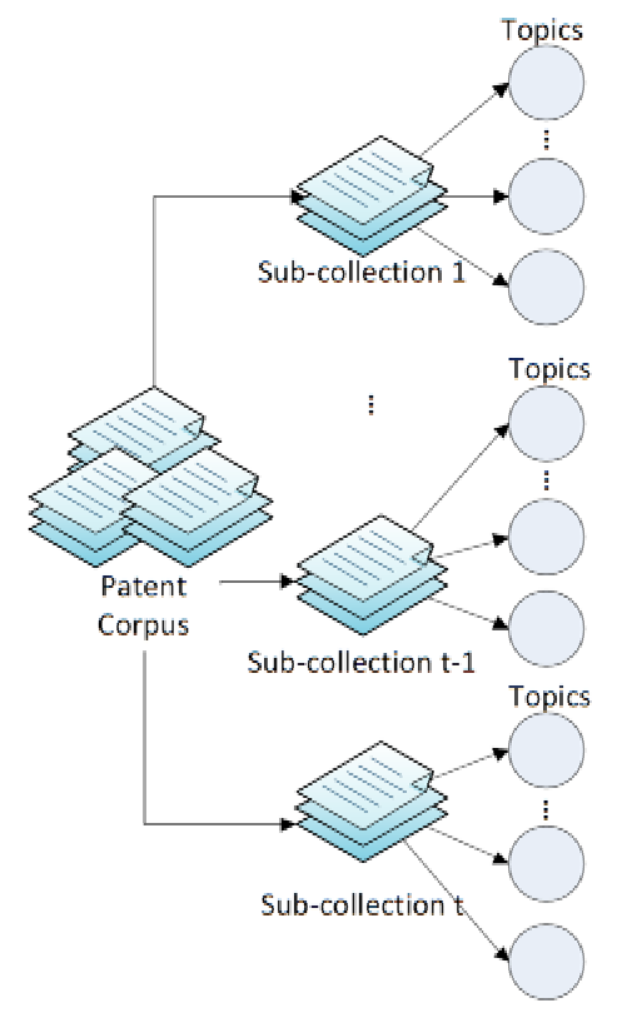

Fig. 3 Relationships between sub-collections and topics

Since we know nothing about the word distributions composing the topics and the topic distributions composing the documents, before topic modeling, assumptions need to be first drawn to determine the parameters $k, \alpha, \beta$ of LDA. According to previous research, hyper-parameters $\alpha, \beta$ of the Dirichlet distribution in LDA have a smoothing effect on multinomial parameters; that is, the lower the values of $\alpha$ and $\beta$ are, the more decisive topic associations there will be [30]. This research sets $\alpha=0.5$ and $\beta=0.1$, which are commonly used in LDA applications [37]. For the setting of $K$, higher $K$ will reduce the topical granularity but increase the processing time. Therefore, during the implementation, $K$ needs to be decided case by case, balancing user requirement and time consumption. Different parameter settings may improve modeling performance, yet optimizing these parameters is beyond the scope of this paper. We then apply Gibbs sampling to infer the needed distributions in LDA. Since the initial values of variables are determined randomly in Gibbs sampling, the outputs of LDA in multiple experiments with a same corpus are slightly different. To ensure the final topic modeling estimation as reliable as possible, evaluation criteria will be needed for the topics finalization.

\section{Final Topics Determination}

As a predefined classification hierarchy built on domain expert judgments, USPC provides a general understanding of the technical domain of concern to one patent, but most of the time, provides only a general understanding. Because patents covering similar topics are usually assigned to a same main USPC, this research uses the main USPC to judge which estimation is closer to the actual topic distribution.

For a sub-collection of corpus, multiple LDA experiments will produce a number of topic assignment matrixes, each indicating the topic distribution proportions of patent documents in the corresponding trial. As shown in Fig. 2, there will be $m$ times experiments for every sub-collection; and after performing each time run, patents in the subcollection are clustered with their calculated topic assignments using the hierarchical clustering approach [38]. Meanwhile, the same group of patents will be also clustered with USPC information. The closer the two clustering results are, the more reliable the topic modeling result is.

Specifically, the values of indexes Jaccard, Folkes \& Mallows and F1 of $m$ times experiments are used to measure the similarity of the clustering results of two groups, one by topics and the other by USPC. The three indices are listed as follows [39]:

$$
\begin{aligned}
& J=a /(a+b+c) \\
& F M=a / \sqrt{r_{1} \cdot r_{2}} \\
& F_{\beta}=\frac{\left(\beta^{2}+1\right) \cdot r_{1} \cdot r_{2}}{\beta^{2} \cdot r_{1}+r_{2}}
\end{aligned}
$$

where $J$ stands for Jaccard coefficient, $F M$ indicates Folkes \& Mallows index, $F_{\beta}$ presents the $\mathrm{F} 1$ indice. In addition, $r_{1}=a /(a+b), r_{2}=a /(a+c)$, where $a$ represents the number of patents that belong to the same cluster of topics and to the same USPC in our case, $b$ is the number of patents that belong to the same cluster of topics but to different USPC, and $c$ is the number of patents that belong to different clusters of topics but to the same USPC. The topic modeling result that provides the highest index values is the optimal one.

\section{E. Topic Change Identification}

After locating the final topics and words underlying the sub-collections of our corpus, we are able to identify the topic change over time. As show in Fig. 4, we compare two groups of topics deriving from different corpus sub-collections, calculating words with a similarity between each topic in $\vec{P}_{i}$ and all the topics in $\vec{P}_{i+1}$. If two topics under different subcollections contain approximately the same group of words, then we believe that these two topics are actually one topic evolving from year to year. However, if there is no similar topic that can be matched in the previous topic collection, then the un-matched topic in the later year is the new one. 


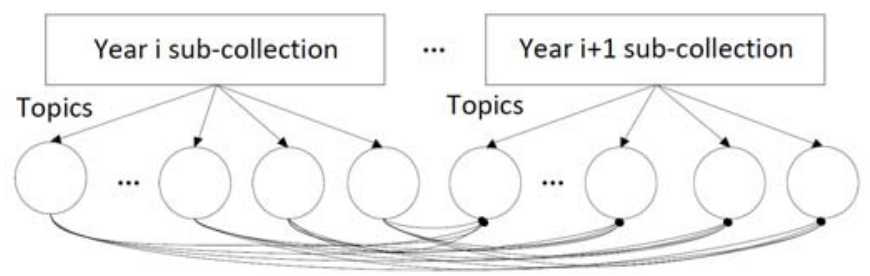

Fig. 4 Topic change identification model

\section{CASE STUDY}

\section{A. Data Collection}

To demonstrate the performance of our proposed approach, patents published during years 2009 to year 2013 in USPTO (http://www.uspto.gov/) with Australia as their assignee country are selected to present a case study. There are 7071 target patents covering 343 different main $\mathrm{USPC}^{12}$. Their patent ID, titles, issue time, inventors, Assignees, United States Patent Classification (USPC), International Patent Classification (IPC) and most importantly, their claims, are clawed from USPTO and placed in a patents tool for further processing. The claims and title for each patent constitute one document in our corpus, which totals 7071 documents on the whole. Then the whole document collection was divided into five sub-collections to present technological feature and essential terms of inventions by Australia assignees in the past five years. The detailed documents number published every year from 2009 to 2010, the term number and USPC number in each corresponding sub-collection are shown in table 1. Although the documents number declined from year 2011, the term number kept rising, which implies that the average complexity of patent claims description is increasing in the resent three years. We also observe that the number of USPC in 2010 had a visible growth, suggesting that there may be a group of new topics appearing in year 2010 .

TABLE 1. THE NUMBER OF DOCUMENTS, TERMS AND USPC OF PATENTS PUBLISHED EACH YEAR

\begin{tabular}{cccc}
\hline Year & Doc NO. & Term NO. & USPC NO. \\
\hline 2009 & 1174 & 19796 & 199 \\
2010 & 1613 & 24726 & 233 \\
2011 & 1746 & 23757 & 228 \\
2012 & 1256 & 25102 & 233 \\
2013 & 1282 & 29714 & 227 \\
\hline
\end{tabular}

\section{B. Topics Determination}

Before topic modeling, as mentioned, a number of parameters need to be set first, including the number of topics, $\alpha, \beta$ of Dirichlet distribution and the number of iterations for Gibbs sampling. In this case study, we applied $K=10$ with model hyper-parameters $\alpha=0.5, \beta=0.1$ to our target documents, to balance the topical granularity, convenience of understanding, and the speed of processing.

\footnotetext{
${ }^{1}$ Data accessed in March 2014

${ }^{2}$ All plant patents are seen as having one same USPC for calculation convenience.
}

Observation for each year were performed $5(m=5)$ runs with 2000 iterations of Gibbs sampling. Indices Folkes \& Mallows, Jaccard, and F1 are calculated after we clustered the patents using both topic assignment and USPC information. The detailed index values of five times experiments are listed in Table 2, where we observe directly that the $3^{\text {rd }}$ experiment (E3) of documents sub-collection in 2009 , the $5^{\text {th }}$ experiment of documents sub-collection in 2010 (E5), the $4^{\text {th }}$ experiment of documents sub-collection in 2011 (E4), the $2^{\text {nd }}$ experiment of documents sub-collection in 2012 and the $3^{\text {rd }}$ experiment (E3) of documents sub-collection in 2013 have the largest value of all three indexes. Thus the topics and parameters provided by these five trials are the final topic modeling result. There are 10 topics describing the essential technological content and feature for each year; and every topic is presented with 10 words given highest probability by this topic.

The topic modeling results are discovered in a completely unsupervised way, with no pre-set classification or domain knowledge assistance. In the past five years, patents owned by Australia assignees cover several important technological topics, such as printhead and nozzle, alkyl compound, pressure apparatus and antibody sequence. The more the topic words are taken into consideration to describe a topic, the more clear and specific the topical semantic meaning will be. Specifically, the topics for each year are presented as follows. The order of the topics is random, and the numbers behind words are the probability values of corresponding topic words. Details of all the topics, the top 10 ranked words and their corresponding probabilities, are shown in the table 1 in the Appendix.

- The topics of year 2009 include printhead (0.0418) cartridge (0.0353), image (0.0217) device (0.0244), ink $(0.0442)$ nozzle $(0.0334)$, composition $(0.0095)$ material $(0.0065)$, portion $(0.0246)$ assembly $(0.0132)$, roller $(0.0142)$ device $(0.0122)$, alkyl $(0.0109)$ compound $(0.0183)$ formula $(0.0111)$, computer $(0.0079)$ gaming (0.0088), signal (0.0278) sensor (0.0108) and antibody $(0.0379)$ sequence $(0.0220)$.

- The topics of year 2010 contain portion (0.0217) assembly (0.0090), light (0.0131)/optical (0.0104) device (0.0104), ink (0.0518) printhead (0.0476), layer (0.0101) material (0.0144), computer $(0.0191)$ memory $(0.0253)$ plurality $(0.0161)$, coded $(0.0252)$ device $(0.0269)$, antibody $(0.0117)$ sequence $(0.0172)$, pressure $(0.0164)$ apparatus (0.0370), alkyl (0.0096) compound (0.0184) and electrode (0.0146) system (0.0175).

- The topics of year 2011 include layer (0.0166) material (0.0188), portion $(0.0260)$ assembly $(0.0202)$, ink (0.0579) printhead (0.0457), acid (0.0201) sequence (0.0234), alkyl (0.0142) compound (0.0159), pressure (0.0161) apparatus (0.0226), light (0.0133) device (0.0114), image (0.0170) print (0.0449), coded (0.0211) device (0.0207) and plurality (0.0084) apparatus (0.0096). 


\begin{tabular}{ccccccc}
\multicolumn{6}{l}{ TABLE 2. INDEXES INFORMATION FOR THE FINAL CHOSEN EXPERIMENT RESULT } \\
\hline Year & Index & $\mathbf{E ~} \mathbf{1}$ & $\mathbf{E} \mathbf{2}$ & $\mathbf{E ~ 3}$ & $\mathbf{E ~ 4}$ & $\mathbf{E ~ 5}$ \\
\hline 2009 & FM & 0.2376 & 0.2803 & $\mathbf{0 . 2 8 4 5}$ & 0.2739 & 0.1948 \\
2009 & DJC & 0.1217 & 0.1500 & $\mathbf{0 . 1 5 0 5}$ & 0.1436 & 0.0962 \\
2009 & F1 & 0.2169 & 0.2608 & $\mathbf{0 . 2 6 1 6}$ & 0.2511 & 0.1755 \\
2010 & FM & 0.2668 & 0.2152 & 0.2253 & 0.3125 & $\mathbf{0 . 3 6 8 8}$ \\
2010 & DJC & 0.1357 & 0.1037 & 0.1077 & 0.1634 & $\mathbf{0 . 2 0 1 7}$ \\
2010 & F1 & 0.2389 & 0.1880 & 0.1944 & 0.2809 & $\mathbf{0 . 3 3 5 6}$ \\
2011 & FM & 0.2521 & 0.2484 & 0.2334 & $\mathbf{0 . 2 6 0 4}$ & 0.2541 \\
2011 & DJC & 0.1334 & 0.1300 & 0.1166 & $\mathbf{0 . 1 3 4 2}$ & 0.1294 \\
2011 & F1 & 0.2354 & 0.2301 & 0.2089 & $\mathbf{0 . 2 3 6 6}$ & 0.2292 \\
2012 & FM & 0.3060 & $\mathbf{0 . 3 2 0 2}$ & 0.2773 & 0.2820 & 0.2686 \\
2012 & DJC & 0.1756 & $\mathbf{0 . 1 8 5 3}$ & 0.1539 & 0.1632 & 0.1521 \\
2012 & F1 & 0.2987 & $\mathbf{0 . 3 1 2 7}$ & 0.2667 & 0.2806 & 0.2640 \\
2013 & FM & 0.2984 & 0.2989 & $\mathbf{0 . 3 3 5 6}$ & 0.3177 & 0.3086 \\
2013 & DJC & 0.1753 & 0.1749 & $\mathbf{0 . 1 9 8 6}$ & 0.1876 & 0.1794 \\
2013 & F1 & 0.2983 & 0.2977 & $\mathbf{0 . 3 3 1 3}$ & 0.3159 & 0.3042 \\
\hline
\end{tabular}

- The topics of year 2012 cover configured (0.0165) signal (0.0325), fluid (0.0209) chamber $(0.0145)$, portion (0.0240) assembly (0.0213), gaming (0.0513) system (0.0205), light (0.0145) lens (0.0067), signal (0.0104) sensor (0.0093), layer (0.0119) material (0.0196), portion $(0.0164)$ apparatus $(0.0101)$, computer $(0.0202)$ memory $(0.0150)$ and acid $(0.0151)$ sequence $(0.0162)$.

- The topics of year 2013 comprise portion (0.0200) assembly (0.0122), gaming (0.0451) controller (0.0226), configured (0.0181) signal (0.0206), cushion (0.0345) mask (0.0287), acid (0.0167) sequence $(0.0158)$, wireless (0.0132) signal (0.0092) sensor (0.0109), layer (0.0120) material (0.0135), optical (0.0095) lens (0.0098), message $(0.0103)$ system $(0.0272)$ and alkyl $(0.0132)$ compound (0.0160).

\section{Topic Change Identification}

After discovering main topics hidden in patent claims of each year, we then use the topic change model to identify the topic variation from years 2009 to 2013. For different groups of topics associated with two consecutive years, we conduct traversal comparison between the topics that belong to the later year with the topics related to the previous year. Topics that contain very similar words are considered as the same topic experiencing innovation; while topics that cannot match any existing ones count as new topics. Fig. 5 illustrates the new topics that arose each year after 2009, by presenting the top 10 words for each topic using Pajek [40].

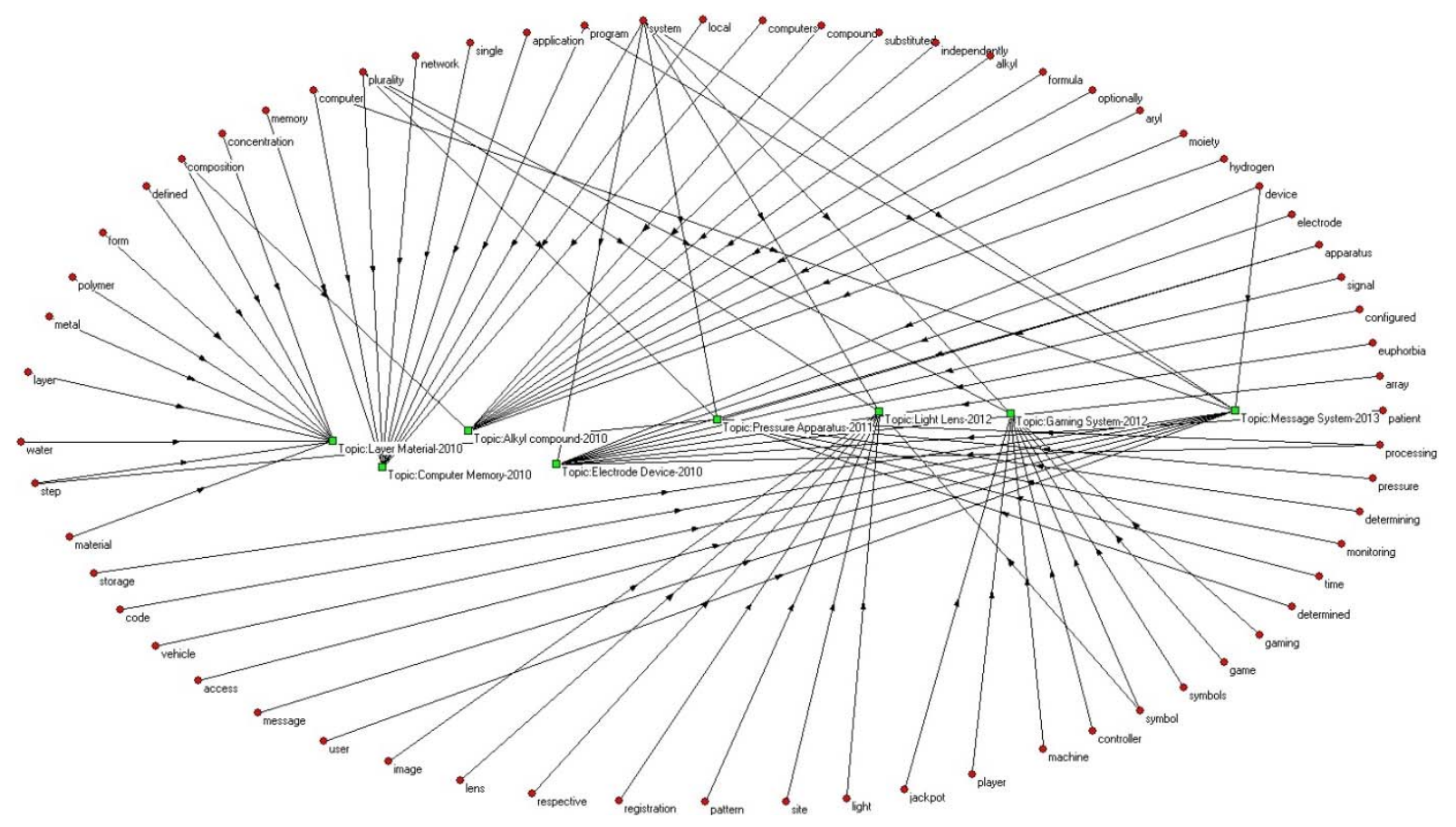

Fig.5 New topics and topmost frequent words of each topic from 2010 to 2013 


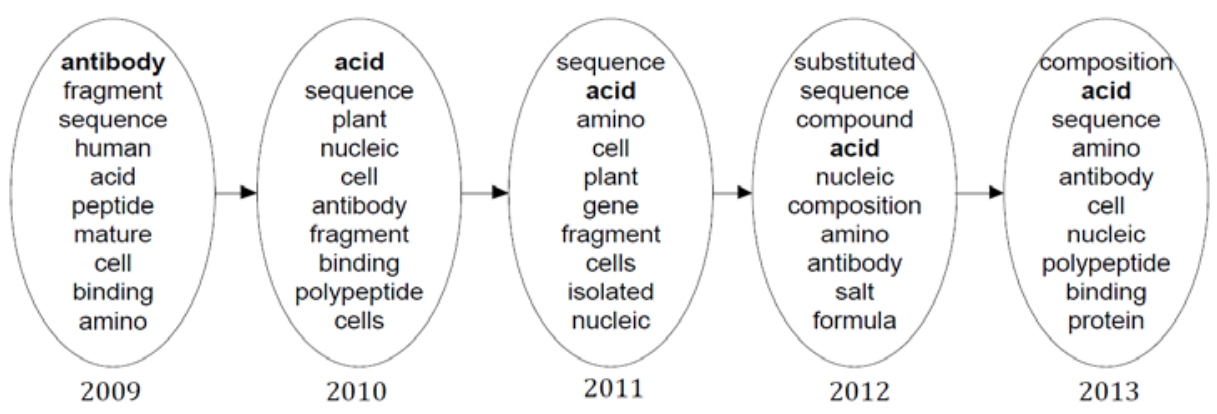

Fig.6 An example of the topic "antibody" evolving over time

In year 2010, four new topics appeared, including layer material that related to metal and polymer composition, electrode device, computer memory and alkyl compound. In year 2011, one new topic appeared, pressure apparatus. Then year 2012 introduced two new topics including light lens and gaming system/controller. Finally, for year 2013, computer system related to vehicle and message appeared as a new topic. All the topics above were identified without assistance of pre-set domain knowledge, which demonstrates the validity of our proposed topic change identification approach. The detailed words and their corresponding probabilities of these new topics are highlighted in boldface in the table 1 of the Appendix.

Moreover, we can also use the proposed approach to discover how the detailed content of a certain topic evolves from year to year. In the case study, topic antibody fragment/sequence is chosen as an example. As shown in Fig. 6 , we observe directly that the word distribution composing the topic develops over time. In year 2009, human and peptide were in the top words list, yet after this, the stress of the topic itself moved to plant, amino acid, nucleic acid and polypeptide. The word 'acid', instead of 'antibody', ranked higher from year 2010 to 2013. The variation of the content of this topic may suggest that, in this area, the key point of technological research and development has shifted to amino/nucleic acid sequence.

\section{CONCLUSION AND FUTURE WORK}

This paper proposed an unsupervised topic change identification approach using Latent Dirichlet Allocation. Patent claims that embody the most significant technological feature and terms are chosen as the main textual data source of our research. To improve the usage of LDA in patent topic extraction, we utilize USPC as a measurement of different estimations, to reduce the randomness effect on the topic modeling. Machine-identified topics are then placed into a topic change model to locate topic variation over time. Since there is no need to define any keywords in advance and all topics are automatically identified in an unsupervised way, this approach is able to set domain experts and analysts free from reading, understanding and summarizing massive technical documents and records. Finally, a case study, using
USPTO patents published during the years 2009 to 2013 with Australia as their assignee country, is presented. The experimental results demonstrate that the proposed approach can be used as an automatic tool to extract topics and identify topic changes from a large volume of patent documents. From the application perspective, the discovered topic variations can be utilized to assist further decision making in R\&D management, especially for newly created innovative enterprises, for example, to provide a full understanding of the topic structure of a certain industry, seek technological opportunities and so on.

As patents and other technological indicators are generating and accumulating in an increasing rate, approaches for automatically identifying topic changes using data mining and machine learning methods will continue to be emphasized. In future work, we will keep focusing on locating topic changes that associate with more meaningful temporal segmentation, like trend turning intervals [41], to identify and analyze the context that contributes to trend changing of patenting activities.

\section{ACKNOWLEDGEMENTS}

The work presented in this paper is partly supported by the Australian Research Council (ARC) under Discovery Project DP140101366 and the National High Technology Research and Development Program of China (Grant No.2014AA015105).

\section{REFERENCES}

[1] Xie, Z. and Miyazaki, K., "Evaluating the effectiveness of keyword search strategy for patent identification," World Patent Information, vol. 35(1), pp. 20-30, 2013.

[2] WIPO. "Patent Cooperation Treaty (PCT) Article 6. Claims," Retrieved 2014, World Intellectual Property Organization, http://www.wipo.int/pct/en/texts/articles/a6.htm.

[3] Campbell, R.S.; "Patent trends as a technological forecasting tool", World Patent Information, vol. 5(3), pp. 137-143, 1983.

[4] Ernst, H.;"The use of patent data for technological forecasting: the diffusion of CNC-technology in the machine tool industry",Small Business Economics,vol.9(4),pp.361-381, 1997.

[5] WIPO, "WIPO Intellectual Property Handbook: Policy, Law and Use. 2 ed", Technological and Legal Developments in Intellectual Property, Vol. 489, pp.17-40, 2004.

[6] Daim, T.U., Kocaoglu, D.F., Anderson, T.R., "Using technological intelligence for strategic decision making in high technology 
environments", Technological Forecasting and Social Change, vol.78(2), pp. 197-198, 2011.

[7] Tseng, Y.H., Lin, C.J., Lin, Y.I., "Text mining techniques for patent analysis", Information Processing \& Management, vol. 43(5), pp. 1216-1247, 2007.

[8] Watts, R.J., Porter, A.L., "Innovation Forecasting", Technological Forecasting and Social Change, vol. 56(1), pp.25-47, 1997.

[9] Yoon, B. and Park, Y., "A systematic approach for identifying technology opportunities: Keyword-based morphology analysis", Technological Forecasting and Social Change, vol. 72(2), pp. 145-160, 2005.

[10] Zhang, Y., Porter, A., Hu, Z., Guo, Y., Newman, N.C., "Term clumping" for technical intelligence: A case study on dye-sensitized solar cells, Technological Forecasting and Social Change, vol. 85(0), pp. 26-39, 2014

[11] Yoon, J. and Kim, K., "TrendPerceptor: A property-function based technology intelligence system for identifying technology trends from patents", Expert Systems with Applications, vol.39(3), pp. 2927-2938, 2012.

[12] Yoon, B., "On the development of a technology intelligence tool for identifying technology opportunity", Expert Systems with Applications, vol. 35(1), pp. 124-135, 2008.

[13] Zhu, D. and Porter, A.L., "Automated extraction and visualization of information for technological intelligence and forecasting", Technological Forecasting and Social Change, vol. 69(5),pp. 495-506, 2002.

[14] Trippe, A.J.; "Patinformatics: Tasks to tools", World Patent Information, vol. 25(3), pp. 211-221, 2003.

[15] Lai, K.K. and Wu, S.J., "Using the patent co-citation approach to establish a new patent classification system", Information Processing \& Management, vol.41(2), pp. 313-330, 2005.

[16] Sheikh, N., Gomez, F. A., Cho, Y.,Siddappa, J., "Forecasting of advanced electronic packaging technologies using bibliometric analysis and Fisher-Pry diffusion model", in Technology Management in the Energy Smart World (PICMET), 2011 Proceedings of PICMET '11, 2011.

[17] Nishijima, Y., Anzai, T., Sengoku, S., Application of bibliometric analysis to market analysis. in Technology Management in the ITDriven Services (PICMET), 2013 Proceedings of PICMET '13, 2013.

[18] Camus, C. and Brancaleon, R., "Intellectual assets management: from patents to knowledge", World Patent Information, vol. 25(2),pp. 155$159,2003$.

[19] Tong, X. and Frame, J.D., "Measuring national technological performance with patent claims data", Research Policy, vol. 23(2), pp. 133-141, 1994.

[20] Yang, S. and Soo, V., "Extract conceptual graphs from plain texts in patent claims", Engineering Applications of Artificial Intelligence, vol. 25(4), pp. 874-887, 2012.

[21] USPTO. "Manual of Patent Examining Procedure: Claim Interpretation. Patent Laws, Regulations, Policies \& Procedures, Chapter 2100, Section 2111”, published 2012, Retrieved 2014, http://www.uspto.gov/web/offices/pac/mpep/s2111.html.

[22] Sheldon, J.G.; How to Write a Patent Application, Practising Law Institute, 1995.
[23] Novelli, E.; "An examination of the antecedents and implications of patent scope", Research Policy, 2014(In press).

[24] USPTO. United States Patent and Trademark Office http://www.uspto.gov/patents/index.jsp.

[25] Blei, D.M., Ng, A.Y., Jordan, M.I., "Latent dirichlet allocation", the Journal of machine Learning research, vol.3, pp. 993-1022, 2003.

[26] Griffiths, T.L. and Steyvers, M., "Finding scientific topics", Proceedings of the National academy of Sciences of the United States of America, vol.101,Suppl 1, pp. 5228-5235, 2004.

[27] Yang, L., Qiu, M.,Gottipati, S.,Zhu, F.,Jiang, J., Sun, H.,Chen, Z., "CQArank: jointly model topics and expertise in community question answering", in Proceedings of the 22nd ACM international conference on Conference on information \& knowledge management, ACM, 2013.

[28] Kim, D. and Oh, A., "Topic Chains for Understanding a News Corpus", in Computational Linguistics and Intelligent Text Processing, A. Gelbukh, Editor, Springer Berlin Heidelberg. pp. 163-176, 2011

[29] Steyvers, M. and Griffiths, T., "Probabilistic topic models", in Latent Semantic Analysis: A road to meaning, D.M. T. Landauer, S. Dennis, and W. Kintsch, Editor, Laurence Erlbaum, 2007.

[30] Heinrich, G.; "Parameter estimation for text analysis", Fraunhofer, IGD: Darmstadt, Germany,2005.

[31] Blei, D.M.;"Probabilistic topic models",Communications of the $A C M$,vol.55(4),pp.77-84, 2012.

[32] Noel, G.E. and Peterson, G.L., "Applicability of Latent Dirichlet Allocation to multi-disk search", Digital Investigation, 2014.

[33] Lukins, S.K., Kraft, N.A., and Etzkorn, L.H., "Bug localization using latent Dirichlet allocation", Information and Software Technology, vol. 52(9), pp. 972-990, 2010.

[34] Lewis,D.D., Yang,Y., Rose,G.T., Li, F., "SMART stopword list", Journal of Machine Learning Research, published 2004, http://jmlr.csail.mit.edu/papers/volume5/lewis04a/a11-smart-stoplist/english.stop.

[35] Wikipedia. Transitional phrase. 2014 http://en.wikipedia.org/wiki/Transitional_phrase.

[36] Haywood,S. AcademicVocabulary, published 2003, Academic Vocabulary - University of Nottingham http://www.nottingham.ac.uk/alzsh3/acvocab/wordlists.htm

[37] Koltcov, S., Koltsova, O., and Nikolenko, S., "Latent dirichlet allocation: stability and applications to studies of user-generated content", in Proceedings of the 2014 ACM conference on Web science, ACM: Bloomington, Indiana, USA, pp. 161-165, 2014.

[38] Steinbach, M., Karypis, G., and Kumar, V., "A comparison of document clustering techniques", in KDD workshop on text mining, Boston. 2000.

[39] Halkidi, M., Batistakis, Y., and Vazirgiannis, M., "On clustering validation techniques", Journal of Intelligent Information Systems, vol. 17(2-3), pp. 107-145, 2001.

[40] Batagelj, V., and Mrvar, A. Pajek - analysis and visualization of large networks Springer, 2004.

[41] Chen, H., Zhang, G., Lu, J., "A patent time series processing component for technology intelligence by trend identification functionality," Neural Computing and Applications, vol.(26:2),pp345353,2015 . 
APPENDIX

TABLE 1. THE TOP 10 RANKED WORDS OF TOPICS FOR YEARS FROM 2009 TO 2013 AND THEIR CORRESPONDING PROBABILITIES

\begin{tabular}{|c|c|c|c|c|c|c|c|c|c|}
\hline \multicolumn{10}{|c|}{ Year 2009} \\
\hline Topic 1 & & Topic 2 & & Topic 3 & & Topic 4 & & Topic 5 & \\
\hline Word & Probability & Word & Probability & Word & Probability & Word & Probability & Word & Probability \\
\hline printhead & 0.0418 & device & 0.0244 & ink & 0.0442 & step & 0.0116 & portion & 0.0246 \\
\hline ink & 0.0353 & image & 0.0217 & ejection & 0.0336 & composition & 0.0095 & body & 0.0150 \\
\hline print & 0.0333 & coded & 0.0209 & nozzle & 0.0334 & gas & 0.0088 & assembly & 0.0132 \\
\hline printer & 0.0252 & system & 0.0195 & inkjet & 0.0307 & leach & 0.0081 & surface & 0.0110 \\
\hline media & 0.0229 & sensing & 0.0181 & printhead & 0.0245 & material & 0.0065 & extending & 0.0092 \\
\hline cartridge & 0.0138 & digital & 0.0132 & drop & 0.0229 & acid & 0.0064 & wall & 0.0091 \\
\hline module & 0.0137 & computer & 0.0105 & apparatus & 0.0224 & fuel & 0.0063 & mask & 0.0081 \\
\hline printing & 0.0135 & camera & 0.0101 & actuator & 0.0220 & water & 0.0059 & adapted & 0.0076 \\
\hline assembly & 0.0132 & identity & 0.0092 & element & 0.0191 & polymer & 0.0058 & substantially & 0.0072 \\
\hline configured & 0.0124 & position & 0.0086 & chamber & 0.0189 & $\mathrm{ph}$ & 0.0055 & support & 0.0069 \\
\hline Topic 6 & & Topic 7 & & Topic 8 & & Topic 9 & & Topic 10 & \\
\hline Word & Probability & Word & Probability & Word & Probability & Word & Probability & Word & Probability \\
\hline support & 0.0152 & compound & 0.0183 & system & 0.0116 & signal & 0.0278 & antibody & 0.0379 \\
\hline roller & 0.0142 & formula & 0.0111 & material & 0.0090 & sensor & 0.0108 & fragment & 0.0246 \\
\hline device & 0.0122 & alkyl & 0.0109 & game & 0.0088 & signals & 0.0107 & sequence & 0.0220 \\
\hline drive & 0.0109 & independently & 0.0102 & plurality & 0.0087 & frequency & 0.0089 & human & 0.0219 \\
\hline assembly & 0.0101 & layer & 0.0098 & computer & 0.0079 & device & 0.0087 & acid & 0.0177 \\
\hline mechanism & 0.0082 & optionally & 0.0095 & gaming & 0.0073 & input & 0.0084 & peptide & 0.0175 \\
\hline surface & 0.0080 & base & 0.0088 & entry & 0.0072 & output & 0.0081 & mature & 0.0164 \\
\hline frame & 0.0075 & detector & 0.0087 & torque & 0.0063 & apparatus & 0.0081 & cell & 0.0157 \\
\hline position & 0.0071 & substituted & 0.0087 & object & 0.0058 & processing & 0.0071 & binding & 0.0138 \\
\hline mounted & 0.0067 & reflector & 0.0087 & service & 0.0054 & power & 0.0067 & amino & 0.0133 \\
\hline \multicolumn{10}{|c|}{ Year 2010} \\
\hline Topic 1 & & Topic 2 & & Topic 3 & & Topic 4 & & Topic 5 & \\
\hline Word & Probability & Word & Probability & Word & Probability & Word & Probability & Word & Probability \\
\hline portion & 0.0217 & signal & 0.0240 & ink & 0.0518 & material & 0.0144 & memory & 0.0253 \\
\hline surface & 0.0126 & light & 0.0131 & printhead & 0.0476 & step & 0.0136 & computer & 0.0191 \\
\hline outer & 0.0095 & system & 0.0121 & nozzle & 0.0214 & water & 0.0101 & plurality & 0.0161 \\
\hline assembly & 0.0090 & optical & 0.0104 & inkjet & 0.0183 & layer & 0.0101 & network & 0.0155 \\
\hline body & 0.0088 & device & 0.0104 & print & 0.0176 & metal & 0.0088 & single & 0.0143 \\
\hline extending & 0.0086 & image & 0.0083 & assembly & 0.0172 & polymer & 0.0081 & application & 0.0141 \\
\hline wall & 0.0080 & power & 0.0076 & printer & 0.0156 & form & 0.0070 & program & 0.0133 \\
\hline support & 0.0076 & frequency & 0.0076 & media & 0.0127 & defined & 0.0067 & system & 0.0117 \\
\hline upper & 0.0073 & output & 0.0069 & ejection & 0.0126 & composition & 0.0066 & local & 0.0103 \\
\hline frame & 0.0071 & sensor & 0.0067 & configured & 0.0110 & concentration & 0.0063 & computers & 0.0097 \\
\hline Topic 6 & & Topic 7 & & Topic 8 & & Topic 9 & & Topic 10 & \\
\hline Word & Probability & Word & Probability & Word & Probability & Word & Probability & Word & Probability \\
\hline device & 0.0269 & acid & 0.0199 & apparatus & 0.0370 & compound & 0.0184 & system & 0.0175 \\
\hline coded & 0.0252 & sequence & 0.0172 & air & 0.0214 & substituted & 0.0183 & device & 0.0154 \\
\hline system & 0.0245 & plant & 0.0159 & pressure & 0.0164 & independently & 0.0140 & electrode & 0.0146 \\
\hline print & 0.0190 & nucleic & 0.0152 & fluid & 0.0148 & alkyl & 0.0096 & apparatus & 0.0107 \\
\hline
\end{tabular}




\begin{tabular}{|c|c|c|c|c|c|c|c|c|c|}
\hline computer & 0.0168 & seq & 0.0146 & valve & 0.0144 & formula & 0.0094 & signal & 0.0105 \\
\hline sensing & 0.0161 & cell & 0.0136 & flow & 0.0140 & optionally & 0.0092 & configured & 0.0095 \\
\hline user & 0.0149 & antibody & 0.0117 & chamber & 0.0131 & aryl & 0.0065 & euphorbia & 0.0095 \\
\hline media & 0.0115 & fragment & 0.0088 & system & 0.0129 & moiety & 0.0051 & array & 0.0079 \\
\hline mobile & 0.0109 & binding & 0.0086 & inlet & 0.0083 & composition & 0.0049 & patient & 0.0074 \\
\hline indicative & 0.0101 & polypeptide & 0.0086 & outlet & 0.0071 & hydrogen & 0.0046 & processing & 0.0071 \\
\hline \multicolumn{10}{|c|}{ Year 2011} \\
\hline Topic 1 & & Topic 2 & & Topic 3 & & Topic 4 & & Topic 5 & \\
\hline Word & Probability & Word & Probability & Word & Probability & Word & Probability & Word & Probability \\
\hline $\begin{array}{l}\text { material } \\
\text { layer } \\
\text { step } \\
\text { composition } \\
\text { range } \\
\text { polymer } \\
\text { coating } \\
\text { metal } \\
\text { solution } \\
\text { forming } \\
\end{array}$ & $\begin{array}{l}0.0188 \\
0.0166 \\
0.0130 \\
0.0083 \\
0.0070 \\
0.0064 \\
0.0060 \\
0.0058 \\
0.0057 \\
0.0056 \\
\end{array}$ & $\begin{array}{l}\text { portion } \\
\text { assembly } \\
\text { mask } \\
\text { support } \\
\text { frame } \\
\text { surface } \\
\text { outer } \\
\text { wall } \\
\text { extending } \\
\text { body } \\
\end{array}$ & $\begin{array}{l}0.0260 \\
0.0202 \\
0.0113 \\
0.0110 \\
0.0105 \\
0.0095 \\
0.0087 \\
0.0084 \\
0.0071 \\
0.0069 \\
\end{array}$ & $\begin{array}{l}\text { ink } \\
\text { printhead } \\
\text { nozzle } \\
\text { inkjet } \\
\text { assembly } \\
\text { chamber } \\
\text { integrated } \\
\text { printer } \\
\text { fluid } \\
\text { plurality } \\
\end{array}$ & $\begin{array}{l}0.0579 \\
0.0457 \\
0.0282 \\
0.0170 \\
0.0163 \\
0.0118 \\
0.0116 \\
0.0113 \\
0.0107 \\
0.0103 \\
\end{array}$ & $\begin{array}{l}\text { sequence } \\
\text { acid } \\
\text { seq } \\
\text { amino } \\
\text { cell } \\
\text { plant } \\
\text { gene } \\
\text { fragment } \\
\text { cells } \\
\text { isolated } \\
\end{array}$ & $\begin{array}{l}0.0234 \\
0.0201 \\
0.0179 \\
0.0138 \\
0.0130 \\
0.0120 \\
0.0113 \\
0.0096 \\
0.0085 \\
0.0084 \\
\end{array}$ & $\begin{array}{l}\text { optionally } \\
\text { substituted } \\
\text { compound } \\
\text { alkyl } \\
\text { lens } \\
\text { independently } \\
\text { optical } \\
\text { aryl } \\
\text { zone } \\
\text { lower } \\
\end{array}$ & $\begin{array}{l}0.0228 \\
0.0224 \\
0.0159 \\
0.0142 \\
0.0102 \\
0.0089 \\
0.0079 \\
0.0074 \\
0.0070 \\
0.0067 \\
\end{array}$ \\
\hline Topic 6 & & Topic 7 & & Topic 8 & & Topic 9 & & Topic 10 & \\
\hline Word & Probability & Word & Probability & Word & Probability & Word & Probability & Word & Probability \\
\hline $\begin{array}{l}\text { apparatus } \\
\text { flow } \\
\text { air } \\
\text { gas } \\
\text { water } \\
\text { pressure } \\
\text { valve } \\
\text { device } \\
\text { fluid } \\
\text { humidifier } \\
\end{array}$ & $\begin{array}{l}0.0226 \\
0.0191 \\
0.0180 \\
0.0180 \\
0.0178 \\
0.0161 \\
0.0158 \\
0.0129 \\
0.0124 \\
0.0110 \\
\end{array}$ & $\begin{array}{l}\text { signal } \\
\text { light } \\
\text { power } \\
\text { device } \\
\text { wireless } \\
\text { apparatus } \\
\text { source } \\
\text { plurality } \\
\text { electrical } \\
\text { optical } \\
\end{array}$ & $\begin{array}{l}0.0203 \\
0.0133 \\
0.0120 \\
0.0114 \\
0.0103 \\
0.0090 \\
0.0090 \\
0.0078 \\
0.0078 \\
0.0074 \\
\end{array}$ & $\begin{array}{l}\text { print } \\
\text { media } \\
\text { printer } \\
\text { image } \\
\text { controller } \\
\text { module } \\
\text { game } \\
\text { gaming } \\
\text { configured } \\
\text { printing } \\
\end{array}$ & $\begin{array}{l}0.0449 \\
0.0296 \\
0.0177 \\
0.0170 \\
0.0148 \\
0.0141 \\
0.0131 \\
0.0129 \\
0.0127 \\
0.0120 \\
\end{array}$ & $\begin{array}{l}\text { system } \\
\text { coded } \\
\text { device } \\
\text { computer } \\
\text { memory } \\
\text { sensing } \\
\text { plurality } \\
\text { identity } \\
\text { indicative } \\
\text { position } \\
\end{array}$ & $\begin{array}{l}0.0289 \\
0.0211 \\
0.0207 \\
0.0186 \\
0.0140 \\
0.0130 \\
0.0114 \\
0.0109 \\
0.0101 \\
0.0086 \\
\end{array}$ & $\begin{array}{l}\text { system } \\
\text { step } \\
\text { apparatus } \\
\text { plurality } \\
\text { pressure } \\
\text { determining } \\
\text { processing } \\
\text { monitoring } \\
\text { time } \\
\text { determined } \\
\end{array}$ & $\begin{array}{l}0.0108 \\
0.0099 \\
0.0096 \\
0.0084 \\
0.0078 \\
0.0076 \\
0.0066 \\
0.0058 \\
0.0057 \\
0.0055 \\
\end{array}$ \\
\hline \multicolumn{10}{|c|}{ Year 2012} \\
\hline Topic 1 & & Topic 2 & & Topic 3 & & Topic 4 & & Topic 5 & \\
\hline Word & Probability & Word & Probability & Word & Probability & Word & Probability & Word & Probability \\
\hline $\begin{array}{l}\text { signal } \\
\text { configured } \\
\text { frequency } \\
\text { optical } \\
\text { sound } \\
\text { system } \\
\text { power } \\
\text { control } \\
\text { electrical } \\
\text { device } \\
\end{array}$ & $\begin{array}{l}0.0325 \\
0.0165 \\
0.0132 \\
0.0116 \\
0.0116 \\
0.0103 \\
0.0092 \\
0.0090 \\
0.0088 \\
0.0087 \\
\end{array}$ & $\begin{array}{l}\text { fluid } \\
\text { gas } \\
\text { flow } \\
\text { chamber } \\
\text { system } \\
\text { valve } \\
\text { water } \\
\text { inlet } \\
\text { pressure } \\
\text { liquid } \\
\end{array}$ & $\begin{array}{l}0.0209 \\
0.0172 \\
0.0151 \\
0.0145 \\
0.0132 \\
0.0129 \\
0.0121 \\
0.0099 \\
0.0097 \\
0.0078 \\
\end{array}$ & $\begin{array}{l}\text { portion } \\
\text { assembly } \\
\text { support } \\
\text { mask } \\
\text { system } \\
\text { element } \\
\text { nasal } \\
\text { adapted } \\
\text { frame } \\
\text { extending } \\
\end{array}$ & $\begin{array}{l}0.0240 \\
0.0213 \\
0.0126 \\
0.0106 \\
0.0087 \\
0.0080 \\
0.0073 \\
0.0072 \\
0.0071 \\
0.0066 \\
\end{array}$ & $\begin{array}{l}\text { gaming } \\
\text { game } \\
\text { system } \\
\text { symbols } \\
\text { symbol } \\
\text { plurality } \\
\text { controller } \\
\text { machine } \\
\text { player } \\
\text { jackpot } \\
\end{array}$ & $\begin{array}{l}0.0513 \\
0.0504 \\
0.0205 \\
0.0190 \\
0.0186 \\
0.0185 \\
0.0172 \\
0.0166 \\
0.0157 \\
0.0127 \\
\end{array}$ & $\begin{array}{l}\text { light } \\
\text { plurality } \\
\text { system } \\
\text { site } \\
\text { pattern } \\
\text { registration } \\
\text { respective } \\
\text { lens } \\
\text { symbol } \\
\text { image } \\
\end{array}$ & $\begin{array}{l}0.0145 \\
0.0114 \\
0.0107 \\
0.0075 \\
0.0070 \\
0.0070 \\
0.0068 \\
0.0067 \\
0.0063 \\
0.0063 \\
\end{array}$ \\
\hline Topic 6 & & Topic 7 & & Topic 8 & & Topic 9 & & Topic 10 & \\
\hline Word & Probability & Word & Probability & Word & Probability & Word & Probability & Word & Probability \\
\hline $\begin{array}{l}\text { time } \\
\text { determining }\end{array}$ & $\begin{array}{l}0.0112 \\
0.0107 \\
\end{array}$ & $\begin{array}{l}\text { material } \\
\text { layer }\end{array}$ & $\begin{array}{l}0.0196 \\
0.0119 \\
\end{array}$ & $\begin{array}{l}\text { portion } \\
\text { apparatus }\end{array}$ & $\begin{array}{l}0.0164 \\
0.0101 \\
\end{array}$ & $\begin{array}{l}\text { system } \\
\text { computer }\end{array}$ & $\begin{array}{l}0.0202 \\
0.0202 \\
\end{array}$ & $\begin{array}{l}\text { substituted } \\
\text { optionally }\end{array}$ & $\begin{array}{l}0.0204 \\
0.0190 \\
\end{array}$ \\
\hline
\end{tabular}




\begin{tabular}{|c|c|c|c|c|c|c|c|c|c|}
\hline signal & 0.0104 & polymer & 0.0100 & surface & 0.0101 & memory & 0.0150 & sequence & 0.0162 \\
\hline test & 0.0093 & metal & 0.0093 & device & 0.0098 & device & 0.0139 & compound & 0.0157 \\
\hline sensor & 0.0093 & surface & 0.0092 & body & 0.0088 & user & 0.0128 & acid & 0.0151 \\
\hline flow & 0.0089 & electrically & 0.0074 & upper & 0.0088 & plurality & 0.0081 & seq & 0.0095 \\
\hline waveform & 0.0085 & step & 0.0067 & extending & 0.0087 & coded & 0.0078 & nucleic & 0.0084 \\
\hline pressure & 0.0085 & conductive & 0.0064 & lower & 0.0081 & content & 0.0078 & composition & 0.0079 \\
\hline predetermined & 0.0070 & cell & 0.0057 & container & 0.0081 & printed & 0.0071 & amino & 0.0072 \\
\hline plant & 0.0068 & component & 0.0056 & assembly & 0.0073 & image & 0.0069 & antibody & 0.0069 \\
\hline \multicolumn{10}{|c|}{ Year 2013} \\
\hline Topic 1 & & Topic 2 & & Topic 3 & & Topic 4 & & Topic 5 & \\
\hline Word & Probability & Word & Probability & Word & Probability & Word & Probability & Word & Probability \\
\hline portion & 0.0200 & game & 0.0555 & signal & 0.0206 & cushion & 0.0345 & composition & 0.0234 \\
\hline assembly & 0.0122 & gaming & 0.0451 & configured & 0.0181 & mask & 0.0287 & seq & 0.0184 \\
\hline body & 0.0107 & symbol & 0.0322 & apparatus & 0.0145 & portion & 0.0285 & acid & 0.0167 \\
\hline surface & 0.0091 & plurality & 0.0274 & device & 0.0139 & assembly & 0.0191 & sequence & 0.0158 \\
\hline extending & 0.0079 & symbols & 0.0238 & stimulation & 0.0105 & frame & 0.0186 & amino & 0.0102 \\
\hline wall & 0.0073 & controller & 0.0226 & signals & 0.0097 & support & 0.0168 & antibody & 0.0091 \\
\hline housing & 0.0072 & player & 0.0189 & system & 0.0096 & structure & 0.0154 & cell & 0.0076 \\
\hline position & 0.0070 & system & 0.0177 & power & 0.0096 & full-face & 0.0124 & nucleic & 0.0071 \\
\hline relative & 0.0063 & arranged & 0.0152 & flow & 0.0091 & nasal & 0.0122 & polypeptide & 0.0068 \\
\hline outer & 0.0062 & machine & 0.0128 & electrical & 0.0086 & underlying & 0.0121 & binding & 0.0066 \\
\hline Topic 6 & & Topic 7 & & Topic 8 & & Topic 9 & & Topic 10 & \\
\hline Word & Probability & Word & Probability & Word & Probability & Word & Probability & Word & Probability \\
\hline device & 0.0286 & material & 0.0135 & image & 0.0236 & system & 0.0272 & substituted & 0.0583 \\
\hline wireless & 0.0132 & layer & 0.0120 & oligonucleotide & 0.0120 & computer & 0.0260 & optionally & 0.0513 \\
\hline system & 0.0115 & fluid & 0.0102 & lens & 0.0098 & user & 0.0154 & compound & 0.0160 \\
\hline plurality & 0.0112 & gas & 0.0094 & optical & 0.0095 & program & 0.0112 & alkyl & 0.0132 \\
\hline sensor & 0.0109 & flow & 0.0084 & antisense & 0.0086 & message & 0.0103 & independently & 0.0129 \\
\hline signal & 0.0092 & water & 0.0083 & light & 0.0085 & access & 0.0088 & formula & 0.0084 \\
\hline processing & 0.0088 & liquid & 0.0081 & plurality & 0.0077 & vehicle & 0.0071 & alkenyl & 0.0084 \\
\hline control & 0.0088 & surface & 0.0075 & system & 0.0070 & code & 0.0061 & salt & 0.0076 \\
\hline devices & 0.0087 & step & 0.0067 & laser & 0.0063 & storage & 0.0060 & alkynyl & 0.0066 \\
\hline component & 0.0082 & electrode & 0.0066 & step & 0.0062 & device & 0.0059 & acceptable & 0.0065 \\
\hline
\end{tabular}

